\title{
Meta
}

Journal des traducteurs

Translators' Journal

BOMATI, YVES, dir. (2008) : L'administration en bons termes.

Paris : Vuibert, 494 p.

\section{François Lavallée}

Volume 56, numéro 1, mars 2011

URI : https://id.erudit.org/iderudit/1003520ar

DOI : https://doi.org/10.7202/1003520ar

Aller au sommaire du numéro

Éditeur(s)

Les Presses de l’Université de Montréal

ISSN

0026-0452 (imprimé)

1492-1421 (numérique)

Découvrir la revue

Citer ce compte rendu

Lavallée, F. (2011). Compte rendu de [Bomati, Yves, dir. (2008) :

L'administration en bons termes. Paris : Vuibert, 494 p.] Meta, 56(1), 218-219.

https://doi.org/10.7202/1003520ar

Ce document est protégé par la loi sur le droit d'auteur. L'utilisation des services d'Érudit (y compris la reproduction) est assujettie à sa politique d'utilisation que vous pouvez consulter en ligne.

https://apropos.erudit.org/fr/usagers/politique-dutilisation/
Cet article est diffusé et préservé par Érudit.

Érudit est un consortium interuniversitaire sans but lucratif composé de l'Université de Montréal, l'Université Laval et l'Université du Québec à Montréal. Il a pour mission la promotion et la valorisation de la recherche. https://www.erudit.org/fr/ 
ogy of translation, an analysis frequently neglected in favour of macro-contextual levels (norms). The last section and book chapter, by Yves Gambier, "Y a-t-il place pour une socio-traductologie?" is explicit in expressing the need to establish and develop 'socio-translation studies,' as he calls this new sub-discipline in the making. In other words, it is a branch that above all must operate around a number of key labels: 'scholars,' 'historiography of the field,' 'institutions and publications shaping and identifying it.'

The challenge has been issued - the book is on sale -, but this pioneering task continues. New proposals, papers, monographs are wanted. Or, as Michaela Wolf states in her enriching "Introduction":

The various thoughts, approaches and elements of theoretical groundwork presented in this introduction are both divergent and competing. However, they all, from varying perspectives and with different methods, aim to foreground the relevance of translation as social practice ... This books aims to show that even if the domain of "translation as a social practice" is still under construction, its outlines are most certainly beginning to come into view (2007: 27, 31).

There is no doubt that Constructing a Sociology of Translation fulfills the major objectives of its editors and contributors. Future researchers will publish new titles on this subject when the discipline is finally 'constructed,' but the impact and vision of this manual will remain. There will be continued gratitute to Michaela Wolf and Alexandra Fukari, its visionary editors, for this monumental achievement.

JUAN ZaRANDONA Universidad de Valladolid, Valladolid, Spain

Bomati, Yves, dir. (2008) : L'administration en bons termes. Paris: Vuibert, $494 \mathrm{p}$.

Quel amoureux de la langue ne s'est jamais perdu en rêveries en feuilletant les citations enchanteresses du Grand Robert (ou, à défaut, du Petit)?

Et pourtant, quel traducteur n'est pas resté perplexe devant une définition ou une citation du Petit Robert qui, certes, le charment, mais n'ont rien pour répondre à ses besoins immédiats, compte tenu de la nature précise des textes qui lui sont confiés?

C'est ainsi que le traducteur se prend parfois à rêver d'un dictionnaire qui, tout en présentant les qualités du Robert, lui parlerait non pas principalement des trésors de la littérature française, mais des ressources du français administratif, celui avec lequel il joue - n'osons pas dire se débat - dans son travail quotidien.
C'est l'outil qu'on lui annonce sur la couverture de L'Administration en bons termes - 1000 mots clés pour comprendre et utiliser le langage administratif. Le traducteur n'aura pas manqué de remarquer le choix des mots comprendre (donc, il y aura des définitions) et utiliser (donc, il y aura des exemples et, si on est chanceux, des cooccurrents).

Or, en feuilletant l'ouvrage, il se rendra compte que celui-ci ne s'adresse pas vraiment au langagier. On le dit d'ailleurs en avant-propos: il s'adresse "aux étudiants et aux fonctionnaires qui passent les concours administratifs, aux fonctionnaires [et] aux citoyens qui désirent dialoguer avec l'administration» (p. 8). La brochette des auteurs est également significative: dix signataires jouissant d'une longue expérience de l'administration sous toutes ses facettes, mais pas de linguiste, et encore moins de traducteur.

Deuxième déception du traducteur québécois - et il s'y sera attendu: l'ouvrage est francofrançais. Partant, une part substantielle de ses articles ne revêtira qu'un intérêt marginal pour lui. Pensons à des termes - nombreux - du genre Fonds régional d'art contemporain (FRAC), Objectif national d'évolution des dépenses d'assurance maladie (ONDAM) ou Direction régionale des affaires culturelles (DRAC). Pour ce qui est des termes à portée plus générale, le traducteur n'apprendra pas nécessairement grand-chose en consultant des articles comme formation permanente, taux d'imposition ou dossier médical. Même dans un article comme État, terme qui n'est pas sans intérêt pour le traducteur administratif qui jongle entre les différentes traductions de government (gouvernement, administration, pouvoirs publics, autorités, ou État?), il n'y trouvera pas une réflexion plus poussée que ce que lui offriront ses documents de référence habituels.

Cela dit, l'ouvrage est loin d'être dénué d'intérêt pour le traducteur curieux de faits de langue idiomatique. En effet, s'il y a un peuple qui s'y connaît en langage administratif, c'est bien les Français. Ainsi, si le traducteur trouvera dans le dictionnaire peu d'articles qui sauront lui être utiles sur le plan strictement du contenu, et si par ailleurs l'ouvrage lui apporte peu ou prou de ces observations linguistiques dont il est friand (distinctions entre quasi-synonymes, cooccurrents), ce n'est pas sans se délecter que le traducteur averti, en examinant les définitions ingénues de ces fonctionnaires, trouvera toutes sortes de tournures propres à l'aider à sortir de ses ornières. Prenons au hasard l'article formalité: «Opération que doivent obligatoirement accomplir l'administration ou l'administré pour assurer la validité de leurs actes ou de leurs demandes. S'agissant de l'autorité administrative, l'inobservation de certaines formalités dites "substantielles" peut 
entraîner l'annulation de l'acte en cause par le juge administratif» (p. 230).

Ainsi, ce n'est pas en cherchant une information précise, mais en butinant dans ce dictionnaire que le traducteur d'ici y trouvera son compte. Par exemple, comment ne pas être intrigué par cette notion de fongibilité d'un budget? ("Caractéristique de crédits budgétaires dont l'objet et la nature ne sont précisés qu'à titre indicatif, le gestionnaire de ces crédits pouvant les redéployer en cours d'exercice sous certaines conditions afin d'optimiser sa gestion» [p. 229]). Encore là, la question se pose: le mot est beau, mais quand sera$\mathrm{t}$-il opportun de l'utiliser? Idem pour infogérance («Technique visant à externaliser tout ou partie de l'informatique et à la confier à un prestataire. » [p. 254]). Une fois encore, c'est peut-être plus la formulation de la définition que son contenu qui sera utile au traducteur.

Au-delà de ces réserves, il y a lieu de signaler une section d'une dizaine de pages par laquelle les auteurs font preuve d'une originalité digne de mention et tombent, cette fois, pile-poil sur les attentes du traducteur. Il s'agit de l'article Verbes de l'administration, qui sort du domaine de prédilection habituel des auteurs de ce genre d'ouvrage - le substantif - pour présenter quelques dizaines de verbes typiques du langage administratif, avec exemples d'utilisation à l'appui et souvent cooccurrents, depuis alléger des charges, arrêter (au sens de /fixer/, /décider/) et asseoir sur (/ calculer sur une base déterminée/) jusqu'à stipuler et surseoir, en passant par fonder en droit, grever, homologuer...

Bref, un outil plein de petits trésors, pour peu qu'on soit conscient des limites inévitables que présente un ouvrage sur le français de l'administration hexagonale pour un traducteur canadien.

François LavalléE Magistrad, Québec, Canada

\section{Antia, BAssey Edem (2007): Indeterminacy in Terminology and LSP. Amsterdam et Phila- delphie: John Bejamins, 233 p.}

Indeterminacy in Terminology and LSP, publié en hommage à Heribert Picht, rassemble des articles de plusieurs auteurs explorant la problématique de l'indétermination selon différentes perspectives. Peu importe leur point de vue, les auteurs s'accordent sur le fait que l'indétermination est un sujet à considérer dans le cadre d'études en terminologie et en langues de spécialité (LSP). L'intérêt pour cette question est relativement récent, car elle va à l'encontre des principes de la terminologie dite classique.
Cet ouvrage est divisé en quatre sections, en fonction du point de vue selon lequel le sujet est abordé. Les articles de la première section traitent de l'indétermination dans une perspective lexicale. Ceux de la deuxième touchent plutôt les aspects épistémologiques de la question. La troisième section concerne les enjeux soulevés par le problème de l'indétermination pour la modélisation en terminologie et en LSP. Dans la dernière section, les contributions d'Heribert Picht en terminologie et en LSP sont exposées.

Dans la première partie, on trouve d'abord l'article de Øivin Andersen, professeur de linguistique en Norvège. Dans son article, il contraste les concepts d'indétermination et de formation adéquate (well-formedness) ainsi que les concepts d'imprécision et d'exactitude. Par la suite, Margaret Rogers, professeure de traduction et de terminologie et directrice du Centre for Terminological Studies de l'Université de Surrey au RoyaumeUni, traite de l'indétermination d'un point de vue lexical dans le contexte de la traduction. Elle y remarque entre autres qu'en traduction, il existe une indétermination dans le réseau d'équivalences entre les unités lexicales participant à la chaîne lexicale, mais que la majorité des paires d'équivalents sont déterminées. Puis, Sergej Griniewicz, professeur de linguistique en Russie, présente une étude dans le domaine de l'anthropologie linguistique. Selon lui, l'indétermination est toujours présente et les changements correspondent aux stades de l'évolution humaine. Anciennement, les limites de la signification des mots n'étaient pas clairement établies, mais ce qui est perçu comme vague aujourd'hui ne l'était probablement pas. Le dernier article de cette partie est de Klaus-Dirk Schmitz, de Cologne en Allemagne. Il porte sur le lien entre l'indétermination et l'évaluation d'un logiciel par l'utilisateur dans une perspective de gestion de la terminologie et de localisation. Il contraste l'imprécision (le référent fait partie du concept) et l'ambiguïté (plus d'une interprétation possible d'un concept).

L'article de Gerhard Budin, qui est notamment professeur et directeur du Département de traduction à l'Université de Vienne, débute la deuxième partie de l'ouvrage. Une perspective épistémologique y est adoptée pour présenter l'évolution du concept de l'indétermination dans certaines disciplines scientifiques. Une importance particulière est accordée à la caractérisation du postmodernisme, notamment à l'aide de la présentation de quelques oppositions. En conclusion, les considérations propres à la terminologie sont présentées. Vient ensuite la contribution de Johan Myking, du Département des langues et de la littérature scandinaves à Bergen en Norvège, qui traite des critiques de la terminologie classique. Ces 